\title{
PROCESSES WITH BLOCK-ASSOCIATED INCREMENTS
}

\author{
ADAM JAKUBOWSKI*** AND \\ JOANNA KARŁOWSKA-PIK, ${ }^{* * * *}$ Nicolaus Copernicus University
}

\begin{abstract}
This paper is motivated by relations between association and independence of random variables. It is well known that, for real random variables, independence implies association in the sense of Esary, Proschan and Walkup (1967), while, for random vectors, this simple relationship breaks. We modify the notion of association in such a way that any vector-valued process with independent increments also has associated increments in the new sense-association between blocks. The new notion is quite natural and admits nice characterization for some classes of processes. In particular, using the covariance interpolation formula due to Houdré, Pérez-Abreu and Surgailis (1998), we show that within the class of multidimensional Gaussian processes, block association of increments is equivalent to supermodularity (in time) of the covariance functions. We also define corresponding versions of weak association, positive association, and negative association. It turns out that the central limit theorem for weakly associated random vectors due to Burton, Dabrowski and Dehling (1986) remains valid, if the weak association is relaxed to the weak association between blocks.
\end{abstract}

Keywords: Association; independence; associated increments; independent increments; weak association; supermodularity

2010 Mathematics Subject Classification: Primary 60G99

Secondary 60G15; 60E07; 60F05

\section{Introduction}

Random variables $X_{1}, X_{2}, \ldots, X_{n}$ are associated if

$$
\operatorname{cov}\left(f\left(X_{1}, X_{2}, \ldots, X_{n}\right), g\left(X_{1}, X_{2}, \ldots, X_{n}\right)\right) \geq 0
$$

for each pair of functions $f, g: \mathbb{R}^{n} \rightarrow \mathbb{R}^{1}$, which are nondecreasing in each coordinate and for which the above covariance exists. This definition, due to Esary et al. [5], seems to be the most appropriate description of positive dependence phenomena encountered in various areas, e.g. reliability theory [1], [13], statistical physics [10], [14], [15], multivariate extremes [19], or random sets [9], to mention but a few. We refer the reader to the recent monograph [2] for properties of association, an extensive list of references, and more abstract formalism of associated random elements.

This paper is motivated by relations between association and independence of random variables. It is well known [2, Theorem 1.8] that any family of independent random variables

Received 14 September 2010; revision received 31 January 2011.

* Postal address: Faculty of Mathematics and Computer Science, Nicolaus Copernicus University, ul. Chopina 12/18, 87-100 Toruń, Poland.

** Email address: adjakubo@mat.umk.pl

*** Email address: joanka@mat.umk.pl 
is associated. In particular, any stochastic process $X=\left\{X_{t}\right\}_{t \geq 0}$ with independent increments also has associated increments in the sense of [6]. The last statement means that, for any choice of sampling points $0<t_{1}<t_{2}<\cdots<t_{n}$, the differences

$$
\Delta_{1} X=X_{t_{1}}-X_{0}, \quad \Delta_{2} X=X_{t_{2}}-X_{t_{1}}, \quad \ldots, \quad \Delta_{n} X=X_{t_{n}}-X_{t_{n-1}}
$$

are associated random variables.

This simple and natural relationship breaks when we pass to processes with values in $\mathbb{R}^{d}, d \geq 2$. Consider, for example, a real process $\left\{Z_{t}\right\}_{t \geq 0}$ with independent increments and nondegenerate marginal laws, and set

$$
Y_{t}=\left[\begin{array}{c}
Z_{t} \\
-Z_{t}
\end{array}\right] .
$$

Then $\left\{Y_{t}\right\}_{t \geq 0}$ retains independent increments, but clearly the components of each increment $\Delta_{k} Y$ (e.g. $\left.X_{1}=Z_{t_{1}}-Z_{0}, X_{2}=-\left(Z_{t_{1}}-Z_{0}\right)\right)$ do not satisfy (1.1); hence, the random vectors $\Delta_{1} Y, \Delta_{2} Y, \ldots, \Delta_{n} Y$ cannot be associated.

We aim at modifying the notion of association in such a way that

- for random variables $(d=1)$, the new notion is equivalent to association;

- any vector-valued process with independent increments also has associated increments in the new sense.

This is done in Section 2, where we introduce association between blocks of random variables. The idea consists in requiring association between real nondecreasing (in each coordinate) functions of blocks. It turns out that the modified notion of association can be easily characterized within classes of random vectors with multivariate normal or infinitely divisible distributions (like the usual association). Similarly, when applied to increments of stochastic processes, the new notion admits nice characterizations within particular classes of processes. For example, for multidimensional Gaussian processes, the block association of increments is equivalent to $L$-superadditivity (or supermodularity) of all covariance functions (see Theorem 3.2). This example shows that association between blocks deals with core properties of multidimensional stochastic processes.

In a similar spirit, in Section 4 we weaken the notions of weak association introduced by Burton et al. [3], positive association (as defined by Bulinski and Shashkin [2]) and negative association (due to Joag-Dev and Proschan [8]). It is interesting that obtained this way 'weak association between blocks' and 'positive association between blocks' coincide while their prototypes differ.

The weak association of random vectors is formally stronger than the weak association between blocks built upon coordinates of vectors. We do not know any example showing that the equality of both classes actually does not hold. On the other hand, an inspection of methods based on factorization of increasing functions and used in the proofs of Theorems 2.1 and 2.2 suggests that verifying whether a sequence of random vectors is 'weakly associated between blocks' may be essentially easier then the corresponding procedure for 'weak association'. Therefore, in Section 5 we restate a complete multidimensional generalization of Newman's central limit theorem [14] and Newman-Wright's invariance principle [16] for sums of stationary associated random variables, originally proved in [3] for weakly associated random vectors. The point is that this result is valid under weak association between blocks, without any change in its proof. 


\section{Association between blocks}

In what follows, when referring to vectors, we mean column vectors.

Let us consider a family $X=\left\{X_{i}, i \in I\right\}$ of real-valued random variables indexed by a finite set $I$. Suppose that $I=\bigcup_{k=1}^{n} I_{k}$, where the sets $I_{k}$ are nonempty and pairwise disjoint. The sets $I_{1}, \ldots, I_{n}$ form the blocks' basis $\mathrm{g}$. Equip each set $I_{k}$ with some arbitrary (but fixed) linear order. Write $X\left(I_{k}\right)$ for the vector with components $\left\{X_{i}, i \in I_{k}\right\}$. Let $|I|$ denote the cardinality of $I$.

We are ready to formulate our basic definition.

Definition 2.1. A family $X=\left\{X_{i}, i \in I\right\}$ is called associated between blocks if, for all nondecreasing functions $f_{k}: \mathbb{R}^{\left|I_{k}\right|} \rightarrow \mathbb{R}, k=1,2, \ldots, n$, the random vector

$$
\left(f_{1}\left(X\left(I_{1}\right)\right), f_{2}\left(X\left(I_{2}\right)\right), \ldots, f_{n}\left(X\left(I_{n}\right)\right)\right)
$$

is associated, i.e. for all nondecreasing functions $g, h: \mathbb{R}^{n} \rightarrow \mathbb{R}$,

$$
\operatorname{cov}\left(g\left(f_{1}\left(X\left(I_{1}\right)\right), \ldots, f_{n}\left(X\left(I_{n}\right)\right)\right), h\left(f_{1}\left(X\left(I_{1}\right)\right), \ldots, f_{n}\left(X\left(I_{n}\right)\right)\right)\right) \geq 0
$$

if the above covariance is well defined.

The very definition and basic properties of association imply the following facts.

Proposition 2.1. Let $X=\left\{X_{i}, i \in I\right\}$ be an associated family of random variables. Then, for an arbitrary partition $I=\bigcup_{k=1}^{n} I_{k}$, we have association of $X$ between blocks based on $I_{1}, \ldots, I_{n}$.

Proposition 2.2. If the vectors $X\left(I_{k}\right), k=1,2, \ldots, n$, are independent then $X$ is associated between blocks.

Proposition 2.3. For a fixed blocks' basis $\mathcal{g}=\left\{I_{1}, I_{2}, \ldots, I_{n}\right\}$, the family $\mathcal{P}_{\mathrm{g}}^{+}$of laws of random vectors which are associated between blocks based on $I_{1}, I_{2}, \ldots, I_{n}$ is closed with respect to the topology of weak convergence.

Let $X=\left\{X_{i}\right\}_{i \in I}$ be an $|I|$-dimensional Gaussian random vector. It is well known [17]but by no means trivial - that the nonnegativity of all entries of the covariance matrix $\Sigma$ of $X$ is necessary and sufficient for association of $X$. We have a very similar situation for the association between blocks.

Theorem 2.1. A Gaussian random vector $X=\left\{X_{i}\right\}_{i \in I}$ is associated between blocks built on $I_{1}, I_{2}, \ldots, I_{n}$ if and only if $\sigma_{k l}=\operatorname{cov}\left(X_{k}, X_{l}\right) \geq 0$ for all $k, l$ which are not in the same block.

While the necessity part in the above theorem is obvious, the sufficiency does not seem to be easy unless advanced tools are used. We propose to exploit the covariance interpolation formula and the technique developed by Houdré et al. [7, Section 2], restated below as Proposition 2.4. Since the covariance formula is valid for general infinitely divisible distributions, Theorem 2.1 is a direct consequence of Theorem 2.2, which will be given after the necessary notation is introduced.

Let $X=\left\{X_{i}\right\}_{i \in I}$ be an $|I|$-dimensional infinitely divisible random vector with the LévyKhinchin triplet $(a, \Sigma, v)$ (we write $X \sim \operatorname{ID}(a, \Sigma, v))$ and the characteristic function $\varphi(t)=$ $\varphi(t ; a, \Sigma, v)$ given by

$$
\ln \varphi(t)=i\langle t, a\rangle-\frac{1}{2}\langle\Sigma t, t\rangle+\int_{\mathbb{R}^{d}}\left(\mathrm{e}^{i\langle t, u\rangle}-1-i\langle t, u\rangle \mathbf{1}_{\{\|u\| \leq 1\}}(u)\right) v(\mathrm{~d} u),
$$


where $\langle\cdot, \cdot\rangle$ is the scalar product in $\mathbb{R}^{|I|}$. Recall that $a \in \mathbb{R}^{|I|}$ is a vector,

$$
\Sigma=\left(\sigma_{k l}\right)_{k, l \in I} \in \mathbb{R}^{|I|} \otimes \mathbb{R}^{|I|}
$$

is the covariance matrix of the Gaussian component of $X$, and $v$ stands for the Lévy measure (for definitions related to infinite divisibility, we refer the reader to [21, Section 8]). We shall associate with $v$ its two-dimensional characteristics $v_{k l}$. If $\pi_{k l}: \mathbb{R}^{|I|} \rightarrow \mathbb{R}^{2}$ are standard projections on $\mathbb{R}^{2}$, i.e.

$$
\pi_{k l}\left(x_{1}, x_{2}, \ldots, x_{|I|}\right)=\left(x_{k}, x_{l}\right), \quad 1 \leq k<l \leq|I|,
$$

we define $v_{k l}$ on $\mathbb{R}^{2}$ by the formula

$$
v_{k l}(A)=\left(v \circ \pi_{k l}^{-1}\right)\left(A \cap\left(\mathbb{R}^{2} \backslash\{0\}\right)\right) .
$$

Note that $v_{k l}$ is a Lévy measure on $\mathbb{R}^{2}$, but it does not have to be the two-dimensional projection of $v$.

A combination of results by Pitt [17] and Resnick [19] states that nonnegativity of all entries of $\Sigma$ together with the concentration of the Lévy measure $v$ on $\left(\mathbb{R}_{+}\right)^{|I|} \cup\left(\mathbb{R}_{-}\right)^{|I|}$ are enough for the association of $X$. Theorem 2.2 establishes analogous conditions for association between blocks of an infinitely divisible random vector.

Theorem 2.2. Let $X \sim \operatorname{ID}(a, \Sigma, v)$. If, for all $k, l \in I$, which are not in the same block,

(i) $\sigma_{k l}$ are nonnegative;

(ii) the measures $v_{k l}$ are concentrated on $\left(\mathbb{R}_{-}\right)^{2} \cup\left(\mathbb{R}_{+}\right)^{2}$,

then $X$ is associated between blocks.

Let $X \sim \operatorname{ID}(a, \Sigma, v)$, and let $\varphi$ be given by (2.1). Define

$$
\varphi_{0}(r, s)=\varphi(r) \varphi(s), \quad \varphi_{1}(r, s)=\varphi(r+s), \quad r, s \in \mathbb{R}^{|I|} .
$$

For each $\alpha \in[0,1]$, let $\left(Y^{\alpha}, Z^{\alpha}\right)$ be an infinitely divisible random vector of dimension $2|I|$ with distribution given by the characteristic function

$$
\varphi_{\alpha}(r, s)=\varphi_{0}^{1-\alpha}(r, s) \varphi_{1}^{\alpha}(r, s)
$$

Then, for each $\alpha \in[0,1]$, we have $Y^{\alpha} \sim Z^{\alpha} \sim X$ and the vector $\left(Y^{\alpha}, Z^{\alpha}\right)$ 'interpolates' between independent copies $Y^{0}, Z^{0}$ of the vector $X$ and the totally dependent copies $Y^{1}=Z^{1}$ of $X$. We are ready to restate the covariance formula due to Houdré et al. [7].

Proposition 2.4. ([7].) For any functions $\psi_{1}, \psi_{2} \in \mathcal{C}_{b}^{1}\left(\mathbb{R}^{|I|}\right)$ (continuously differentiable with bounded derivatives),

$$
\begin{aligned}
\operatorname{cov}\left(\psi_{1}(X), \psi_{2}(X)\right)=\int_{0}^{1} \mathrm{E}( & \left\langle\Sigma \nabla \psi_{1}\left(Y^{\alpha}\right), \nabla \psi_{2}\left(Z^{\alpha}\right)\right\rangle \\
& \left.+\int_{\mathbb{R}^{|I|}} \Delta_{u} \psi_{1}\left(Y^{\alpha}\right) \Delta_{u} \psi_{2}\left(Z^{\alpha}\right) v(\mathrm{~d} u)\right) \mathrm{d} \alpha
\end{aligned}
$$

where $\nabla$ is the gradient operator and $\Delta_{u} \psi(x)=\psi(x+u)-\psi(x)$. 
Now we can turn to the proof of Theorem 2.2, keeping in mind that it is enough to study (1.1) only for functions from $C_{b}^{1}\left(\mathbb{R}^{|I|}\right)$ (see, e.g. [2, Theorem 1.5]).

Proof of Theorem 2.2. Choose nondecreasing and $C_{b}^{1}$ functions $f_{i}: \mathbb{R}^{\left|I_{i}\right|} \rightarrow \mathbb{R}, k=1,2$, $\ldots, n$, and denote by $F$ the mapping from $\mathbb{R}^{|I|}$ into $\mathbb{R}^{n}$ given by

$$
F\left(x_{k}, k \in I\right)=\left(f_{1}\left(x_{k}, k \in I_{1}\right), \ldots, f_{n}\left(x_{k}, k \in I_{n}\right)\right) .
$$

We will identify the functions $f_{i}$ with their corresponding extensions $\tilde{f}_{i}(x)=f_{i}\left(\pi_{I_{i}}(x)\right)$.

Let $g, h: \mathbb{R}^{n} \rightarrow \mathbb{R}$ be nondecreasing and $C_{b}^{1}$. Our goal is to establish the sign of the covariance

$$
\begin{aligned}
\operatorname{cov}(g(F(X)), h(F(X)))=\int_{0}^{1} \mathrm{E}( & \left\langle\Sigma \nabla(g \circ F)\left(Y^{\alpha}\right), \nabla(h \circ F)\left(Z^{\alpha}\right)\right\rangle \\
& \left.+\int_{\mathbb{R}^{|I|}} \Delta_{u}(g \circ F)\left(Y^{\alpha}\right) \Delta_{u}(h \circ F)\left(Z^{\alpha}\right) v(\mathrm{~d} u)\right) \mathrm{d} \alpha .
\end{aligned}
$$

Applying the chain rule we find that $\nabla(g \circ F)(y)$ is the product of the transposed matrix of partial derivatives of $F$ and the vector $(\nabla g)(F(y))$. The first of these factors is the matrix with $n$ columns and $|I|$ rows, with nonzero elements only for $k \in I_{i}$ ( $i$ is the column and $k$ is the row number). So

$$
(\nabla(g \circ F)(y))_{k}= \begin{cases}\frac{\partial f_{i}}{\partial x_{k}}(y) \frac{\partial g}{\partial v_{i}}(F(y)) & \text { if } k \in I_{i}, i=1,2, \ldots, n, \\ 0 & \text { otherwise. }\end{cases}
$$

Hence, the scalar product in the covariance formula has the following form:

$$
\begin{aligned}
\langle\Sigma \nabla(g \circ F)(y), \nabla(h \circ F)(z)\rangle & \\
= & \sum_{i=1}^{n} \sum_{j=1}^{n} \sum_{k \in I_{i}} \sum_{l \in I_{j}} \sigma_{k l} \frac{\partial f_{i}}{\partial x_{k}}(y) \frac{\partial g}{\partial v_{i}}(F(y)) \frac{\partial f_{j}}{\partial x_{l}}(z) \frac{\partial h}{\partial v_{j}}(F(z)) \\
= & \sum_{i=1}^{n} \frac{\partial g}{\partial v_{i}}(F(y)) \frac{\partial h}{\partial v_{i}}(F(z))\left(\sum_{k \in I_{i}} \sum_{l \in I_{i}} \sigma_{k l} \frac{\partial f_{i}}{\partial x_{k}}(y) \frac{\partial f_{i}}{\partial x_{l}}(z)\right) \\
& +\sum_{i=1}^{n} \sum_{\substack{j=1 \\
j \neq i}}^{n} \sum_{k \in I_{i}} \sum_{l \in I_{j}} \sigma_{k l} \frac{\partial f_{i}}{\partial x_{k}}(y) \frac{\partial g}{\partial v_{i}}(F(y)) \frac{\partial f_{j}}{\partial x_{l}}(z) \frac{\partial h}{\partial v_{j}}(F(z)) .
\end{aligned}
$$

The expression in line (2.4) is nonnegative because the partial derivatives are nonnegative and

$$
\sum_{k \in I_{i}} \sum_{l \in I_{i}} \sigma_{k l} \frac{\partial f_{i}}{\partial x_{k}}(y) \frac{\partial f_{i}}{\partial x_{l}}(z) \geq 0
$$

due to the fact that $\sigma_{k l}$ for $k, l \in I_{i}$ are entries of the covariance matrix of the vector $X\left(I_{i}\right)$. The expression in line (2.5) is nonnegative because all partial derivatives are nonnegative and $\sigma_{k l} \geq 0$ if $k, l$ are not in the same block.

It remains to check that the second summand in (2.3) is nonnegative. Let us consider the following sets:

$$
\begin{aligned}
& A_{+}=\{u: F(y+u) \geq F(y)\} \cap\{u: F(z+u) \geq F(z)\}, \\
& A_{-}=\{u: F(y+u) \leq F(y)\} \cap\{u: F(z+u) \leq F(z)\} .
\end{aligned}
$$


It is easy to see that, on the set $A=A_{+} \cup A_{-}$,

$$
\Delta_{u}(g \circ F)(y) \Delta_{u}(h \circ F)(z)=(g(F(y+u))-g(F(y)))(h(F(z+u))-h(F(z))) \geq 0,
$$

for both factors are at the same time either nonnegative or nonpositive. It follows that it is enough to prove that

$$
v\left(A^{\mathrm{c}}\right)=v\left(A_{+}^{\mathrm{c}} \cap A_{-}^{\mathrm{c}}\right)=0,
$$

where $B^{\mathrm{c}}$ is the complement of $B$. We have

$$
A_{+}=\bigcap_{i=1}^{n}\left\{u: f_{i}(y+u) \geq f_{i}(y), f_{i}(z+u) \geq f_{i}(z)\right\}
$$

hence,

$$
A_{+}^{\mathrm{c}}=\bigcup_{i=1}^{n}\left\{u: f_{i}(y+u)<f_{i}(y)\right\} \cup\left\{u: f_{i}(z+u)<f_{i}(z)\right\},
$$

and, similarly,

$$
A_{-}^{\mathrm{c}}=\bigcup_{j=1}^{n}\left\{u: f_{j}(y+u)>f_{j}(y)\right\} \cup\left\{u: f_{j}(z+u)>f_{j}(z)\right\} .
$$

So $A^{\mathrm{c}}=\bigcup_{1 \leq i \neq j \leq n}^{n} B_{i j}$, where

$$
\begin{aligned}
B_{i j}= & \left\{u: f_{i}(y+u)<f_{i}(y), f_{j}(y+u)>f_{j}(y)\right\} \\
& \cup\left\{u: f_{i}(y+u)<f_{i}(y), f_{j}(z+u)>f_{j}(z)\right\} \\
& \cup\left\{u: f_{i}(z+u)<f_{i}(z), f_{j}(y+u)>f_{j}(y)\right\} \\
& \cup\left\{u: f_{i}(z+u)<f_{i}(z), f_{j}(z+u)>f_{j}(z)\right\} .
\end{aligned}
$$

Since the $f_{i}$ s are nondecreasing, $f_{i}(x+u)<f_{i}(x)$ implies that there exists $k \in I_{i}$ such that $u_{k}<0$. (If $u$ were in $\left(\mathbb{R}_{+}\right)^{I_{i}}$, we would have $f_{i}(x+u) \geq f_{i}(x)$.) Similarly, $f_{i}(x+u)>f_{i}(x)$ implies that there exists $l \in I_{i}$ such that $u_{l}>0$. Thus, we obtain

$$
B_{i j} \subset \bigcup_{k \in I_{i}} \bigcup_{l \in I_{j}}\left\{u: u_{k}<0, u_{l}>0\right\} .
$$

But $i \neq j$ and so $k$ and $l$ in the above union of sets are not in the same block. It follows that

$$
v\left(\left\{u: u_{k}<0, u_{l}>0\right\}\right)=v_{k l}((-\infty, 0) \times(0,+\infty))=0 .
$$

Hence, $v\left(B_{i j}\right)=0$ and $v\left(A^{\mathrm{c}}\right)=0$.

For future purposes, we need a convenient reformulation of the condition imposed in Theorem 2.2 on the two-dimensional Lévy measures $v_{k l}$.

Proposition 2.5. Let $v$ be a measure on $\mathbb{R}^{|I|}$, and let the measures $v_{k l}$ on $\mathbb{R}^{2}$ be defined by (2.2). Then the following statements are equivalent.

(i) For all $k, l \in I$, which are not in the same block, the measures $v_{k l}$ are concentrated on $\left(\mathbb{R}_{-}\right)^{2} \cup\left(\mathbb{R}_{+}\right)^{2}$. 
(ii) The measure $v$ is concentrated on the set

$$
S=\left(\mathbb{R}_{+}\right)^{|I|} \cup\left(\mathbb{R}_{-}\right)^{|I|} \cup U,
$$

where

$$
U=\bigcup_{m=1}^{n}\left(\{0\}^{\sum_{i=1}^{m-1}\left|I_{i}\right|} \times \mathbb{R}^{\left|I_{m}\right|} \times\{0\}^{\sum_{j=m+1}^{n}\left|I_{j}\right|}\right) .
$$

Proof. It is clear that if $v$ concentrates on $S$ given in (2.6) then the $v_{k l}$ satisfy (i). Thus, we have to prove the implication (i) $\Rightarrow$ (ii) only. For notational convenience, let us write $k \sim l$ if $k$ and $l$ are in the same block and $k \ngtr l$ otherwise. Let us also define $D_{k}^{+}=\left\{x \in \mathbb{R}^{|I|}: x_{k}>0\right\}$, $D_{k}^{-}=\left\{x \in \mathbb{R}^{|I|}: x_{k}<0\right\}$, and

$$
D=\bigcup_{\{(k, l): k \neq l\}} D_{k}^{+} \cap D_{l}^{-} .
$$

Then (i) implies that $v\left(D_{k}^{+} \cap D_{l}^{-}\right)=0$ for all pairs $(k, l)$ such that $k \nsim l$ and so

$$
v(D)=0 .
$$

Now (ii) follows from (2.6), (2.7), and the observation that

$$
\mathbb{R}^{|I|}=\left(\mathbb{R}_{+}\right)^{|I|} \cup\left(\mathbb{R}_{-}\right)^{|I|} \cup U \cup D=S \cup D .
$$

The example given by Samorodnitsky [20] shows that there exists an associated (so associated between blocks of the length 1 , too) random vector with two-dimensional infinitely divisible distribution and with Lévy measure assigning a positive mass out of the set $\left(\mathbb{R}_{+}\right)^{2} \cup\left(\mathbb{R}_{-}\right)^{2}$. So in Theorem 2.2 the condition related to the concentration of the measures $v_{k l}$ is not necessary for association between blocks of the multidimensional vector with infinitely divisible distribution.

On the other hand, there exists a natural framework proposed by Samorodnitsky [20] in which the concentration of the Lévy measure on $\left(\mathbb{R}_{+}\right)^{|I|} \cup\left(\mathbb{R}_{-}\right)^{|I|}$ is necessary. The theorem below can be proved in much the same way as Theorem 3.1 of [20] or Proposition 3 of [7].

Theorem 2.3. Let $X \sim \operatorname{ID}(a, \Sigma, v)$. Let $\left\{X_{t}, t \geq 0\right\}$ be a Lévy process with $X_{1} \stackrel{\mathrm{D}}{=} X$. Then the following statements are equivalent.

(i) For every $t>0$ and any choice of nondecreasing functions $f_{1}: \mathbb{R}^{\left|I_{1}\right|} \rightarrow \mathbb{R}, \ldots, f_{n}$ : $\mathbb{R}^{\left|I_{n}\right|} \rightarrow \mathbb{R}$, the vector

$$
\left(f_{1}\left(\left(X_{t}\right)_{I_{1}}\right), \ldots, f_{n}\left(\left(X_{t}\right)_{I_{n}}\right)\right)
$$

is associated.

(ii) For all indices $k, l$ which are not in the same block, the entries $\sigma_{k l}$ of the matrix $\Sigma$ are nonnegative and the Lévy measures $v_{k l}$ concentrate on the set $\left(\mathbb{R}_{+}\right)^{2} \cup\left(\mathbb{R}_{-}\right)^{2}$.

\section{Block association of increments of stochastic processes}

Let $\left\{X_{t}=\left(X_{t}^{1}, X_{t}^{2}, \ldots, X_{t}^{d}\right), t \in \mathbb{R}\right\}$ be a $d$-dimensional stochastic process, and let $0<$ $t_{1}<t_{2}<\cdots<t_{n}$. We can consider an $n d$-dimensional random vector formed by the increments

$$
X_{t_{1}}-X_{0}, \quad X_{t_{2}}-X_{t_{1}}, \quad \ldots, \quad X_{t_{n}}-X_{t_{n-1}} .
$$

Such a vector has naturally distinguished blocks of the length $d$. The first is formed by the components of $X_{t_{1}}-X_{0}$, the second by the components of $X_{t_{2}}-X_{t_{1}}$, and so on. Hence, according to Definition 2.1, we have the following. 
Definition 3.1. A $d$-dimensional stochastic process $\left\{X_{t}, t \in \mathbb{R}\right\}$ has block-associated increments if, for every $n \in \mathbb{N}$ and any choice of $0<t_{1}<t_{2}<\cdots<t_{n}$, the increments

$$
X_{t_{1}}-X_{0}, \quad X_{t_{2}}-X_{t_{1}}, \quad \ldots, \quad X_{t_{n}}-X_{t_{n-1}}
$$

form the vector associated between blocks.

With such a definition we have the expected result.

Theorem 3.1. Every process with independent increments has block-associated increments.

Next we shall discuss Gaussian processes.

Theorem 3.2. Let $\left\{X_{t}, t \geq 0\right\}$ be a d-dimensional Gaussian process with the covariance functions $K^{k, l}(s, t)=\operatorname{cov}\left(X_{s}^{k}, X_{t}^{l}\right), k, l=1, \ldots, d$. The process $\left\{X_{t}, t \geq 0\right\}$ has blockassociated increments if and only if its covariance functions are L-superadditive on $\{(s, t)$; $s \leq t\}$, i.e.

$$
K^{k, l}\left(s_{1}, t_{1}\right)-K^{k, l}\left(s_{2}, t_{1}\right)-K^{k, l}\left(s_{1}, t_{2}\right)+K^{k, l}\left(s_{2}, t_{2}\right) \geq 0
$$

for all $0 \leq s_{1} \leq s_{2} \leq t_{1} \leq t_{2}$.

Proof. Let us consider the $n d$-dimensional vector

$$
\left(X_{t_{1}}^{1}-X_{0}^{1}, \ldots, X_{t_{1}}^{d}-X_{0}^{d}, \ldots, X_{t_{n}}^{1}-X_{t_{n-1}}^{1}, \ldots, X_{t_{n}}^{d}-X_{t_{n-1}}^{d}\right),
$$

where $0<t_{1}<t_{2}<\cdots<t_{n}$. As we know from Theorem 2.1, the process $\left\{X_{t}, t \geq 0\right\}$ has block-associated increments if and only if, for all $k, l=1, \ldots, d$, and $1 \leq i<j \leq n, i \neq j$, the covariances

are nonnegative. But

$$
\sigma_{i j}^{k, l}=\operatorname{cov}\left(X_{t_{i}}^{k}-X_{t_{i-1}}^{k}, X_{t_{j}}^{l}-X_{t_{j-1}}^{l}\right)
$$

$$
\begin{aligned}
0 \leq \sigma_{i j}^{k, l} & =\operatorname{cov}\left(X_{t_{i}}^{k}-X_{t_{i-1}}^{k}, X_{t_{j}}^{l}-X_{t_{j-1}}^{l}\right) \\
& =K^{k, l}\left(t_{i}, t_{j}\right)-K^{k, l}\left(t_{i}, t_{j-1}\right)-K^{k, l}\left(t_{i-1}, t_{j}\right)+K^{k, l}\left(t_{i-1}, t_{j-1}\right) .
\end{aligned}
$$

Remark 3.1. The notion of $L$-superadditivity is well known; see, for example, [11, Chapter 6, Section D].

Corollary 3.1. If the covariance functions $K^{k, l}(k, l=1, \ldots, d)$ of the d-dimensional Gaussian process $\left\{X_{t}, t \geq 0\right\}$ are continuously twice differentiable for $s \neq t$, then $\left\{X_{t}, t \geq 0\right\}$ has block-associated increments if and only if

$$
\frac{\partial^{2}}{\partial s \partial t} K^{k, l}(s, t) \geq 0 \text { for } s \neq t \text { and } k, l=1,2, \ldots, d .
$$

Proof. The $L$-superadditivity of the covariance functions is, under the corollary's assumptions, equivalent to the nonnegativity of the mixed second derivatives. Indeed,

$$
\begin{gathered}
K^{k, l}\left(t_{i}, t_{j}\right)-K^{k, l}\left(t_{i}, t_{j-1}\right)-K^{k, l}\left(t_{i-1}, t_{j}\right)+K^{k, l}\left(t_{i-1}, t_{j-1}\right) \\
=\int_{t_{i-1}}^{t_{i}}\left(\frac{\partial K^{k, l}}{\partial u}\left(u, t_{j}\right)-\frac{\partial K^{k, l}}{\partial u}\left(u, t_{j-1}\right)\right) \mathrm{d} u \\
=\int_{t_{i-1}}^{t_{i}} \int_{t_{j-1}}^{t_{j}} \frac{\partial^{2} K^{k, l}}{\partial v \partial u}(u, v) \mathrm{d} v \mathrm{~d} u,
\end{gathered}
$$

and $\left(t_{i-1}, t_{i}\right)$ and $\left(t_{j-1}, t_{j}\right)$ are arbitrary disjoint intervals in $(0,+\infty)$. 
Similarly as Theorem 2.1 produced Theorem 3.2, we could also use Theorem 2.2 for writing a corresponding result for infinitely divisible processes (processes with infinitely divisible, finitely dimensional distributions—-see, e.g. [12] or [18]). We shall do that in a special case and using Proposition 2.5.

Theorem 3.3. Let $\left\{X_{t}, t \geq 0\right\}$ be a d-dimensional infinitely divisible stochastic process. Let us suppose that, for every choice of $0=t_{0}<t_{1}<t_{2}<\cdots<t_{n}$, the distribution of $\left(X_{0}, X_{t_{1}}, X_{t_{2}}, \ldots, X_{t_{n}}\right)$ does not have the Gaussian component and that the support of its Lévy measure $v_{0, t_{1}, \ldots, t_{n}}$ is contained in the set

$$
\begin{aligned}
\left\{\left(x_{0}, x_{1}, \ldots, x_{n}\right):\right. & x_{0} \leq x_{1} \leq x_{2} \leq \cdots \leq x_{n} \text { or } x_{0} \geq x_{1} \geq x_{2} \geq \cdots \geq x_{n} \\
& \text { or } x_{1}=x_{2}=\cdots=x_{n} \text { or, for some } m=2,3, \ldots, n, \\
& \left.x_{0}=x_{1}=\cdots=x_{m-1}, x_{m}=x_{m+1}=\cdots=x_{n}\right\}
\end{aligned}
$$

where $x_{0}, x_{1}, x_{2}, \ldots, x_{n}$ are $d$-dimensional vectors and ' $\leq$ ' and ' $\geq$ ' are coordinatewise inequalities.

Then $\left\{X_{t}, t \geq 0\right\}$ has block-associated increments.

Proof. Let $U: \mathbb{R}^{(n+1) d} \rightarrow \mathbb{R}^{n d}$ be given by

$$
U(x)=U\left(x_{0}, x_{1}, \ldots, x_{n}\right)=\left(x_{1}-x_{0}, x_{2}-x_{1}, \ldots, x_{n}-x_{n-1}\right) .
$$

It is well known that if $\left(X_{0}, X_{t_{1}}, X_{t_{2}}, \ldots, X_{t_{n}}\right)$ has an infinitely divisible distribution with a Lévy measure $v_{0, t_{1}, \ldots, t_{n}}$ then the vector of increments $\left(X_{t_{1}}-X_{0}, X_{t_{2}}-X_{t_{1}}, \ldots, X_{t_{n}}-X_{t_{n-1}}\right)$ also has an infinitely divisible distribution with the Lévy measure $v_{0, t_{1}, \ldots, t_{n}} \circ U^{-1}$ (up to an atom at 0 ; see, e.g. [21, Proposition 11.10]). For the block association of increments, it is enough that the Lévy measures $\nu_{0, t_{1}, \ldots, t_{n}} \circ U^{-1}$ concentrate on $S=\left(\mathbb{R}_{+}\right)^{n d} \cup\left(\mathbb{R}_{-}\right)^{n d} \cup \bigcup_{m=1}^{n}\left(\{0\}^{(m-1) d} \times\right.$ $\mathbb{R}^{d} \times\{0\}^{(n-m) d}$ ) (Proposition 2.5), so, for $\nu_{0, t_{1}, \ldots, t_{n}}$, it is enough to concentrate on the union of sets

$$
\begin{aligned}
& \left\{x: x_{1}-x_{0} \geq 0, x_{2}-x_{1} \geq 0, \ldots, x_{n}-x_{n-1} \geq 0\right\} \\
& \cup\left\{x: x_{1}-x_{0} \leq 0, x_{2}-x_{1} \leq 0, \ldots, x_{n}-x_{n-1} \leq 0\right\} \\
& \cup\left\{x: x_{2}-x_{1}=0, \ldots, x_{n}-x_{n-1}=0\right\} \\
& \cup \bigcup_{m=2}^{n}\left\{x: x_{1}-x_{0}=0, \ldots, x_{m-1}-x_{m-2}=0\right\} \\
& \quad \cap\left\{x: x_{m+1}-x_{m}=0, \ldots, x_{n}-x_{n-1}=0\right\},
\end{aligned}
$$

which equals

$$
\begin{gathered}
\left\{x: x_{0} \leq x_{1} \leq x_{2} \leq \cdots \leq x_{n} \text { or } x_{0} \geq x_{1} \geq x_{2} \geq \cdots \geq x_{n}\right. \\
\quad \text { or } x_{1}=x_{2}=\cdots=x_{n} \text { or, for some } m=2, \ldots, n, \\
\\
\left.x_{0}=x_{1}=\cdots=x_{m-1}, x_{m}=x_{m+1}=\cdots=x_{n}\right\} .
\end{gathered}
$$

Remark 3.2. It is clear that the finite-dimensional properties of the Lévy measures $v_{t_{0}, t_{1}, \ldots, t_{n}}$ can be expressed in terms of their projective limit $v$ (see [12]): $v$ must be concentrated on the union of sets consisting of nondecreasing trajectories, nonincreasing trajectories, and rather mysterious trajectories admitting only one jump. 


\section{Some other notions of relaxed association}

The following notion was introduced by Burton et al. [3].

Definition 4.1. A sequence of $d$-dimensional random vectors $\left(X_{1}, X_{2}, \ldots, X_{m}\right)$ is said to be weakly associated if whenever $\pi$ is a permutation of $\{1,2, \ldots, m\}, 1 \leq k<m$, and $g: \mathbb{R}^{k d} \rightarrow$ $\mathbb{R}$ and $h: \mathbb{R}^{(m-k) d} \rightarrow \mathbb{R}$ are coordinatewise nondecreasing, then

$$
\operatorname{cov}\left(g\left(X_{\pi(1)}, X_{\pi(2)}, \ldots, X_{\pi(k)}\right), h\left(X_{\pi(k+1)}, X_{\pi(k+2)}, \ldots, X_{\pi(m)}\right)\right) \geq 0,
$$

if the covariance exists. A family of random vectors is weakly associated if its every finite subfamily is weakly associated.

Burton et al. [3, Theorem 1] provided an example of a sequence of weakly associated random variables $(d=1)$, which are not associated. Let $Y_{1}, Y_{2}, \ldots$ be such a sequence. Fix $d>1$, and define a sequence of $d$-dimensional random vectors by

$$
X_{k}=(\underbrace{Y_{k}, Y_{k}, \ldots, Y_{k}}_{k \text { times }}) \text {. }
$$

Then it is easy to see that $X_{1}, X_{2}, \ldots$ is weakly associated but it is not associated between blocks built upon coordinates. The following definition is in the spirit of Section 2.

Definition 4.2. A family $X=\left\{X_{i}, i \in I\right\}$ is called weakly associated between blocks if, for all nondecreasing functions $f_{k}: \mathbb{R}^{\left|I_{k}\right|} \rightarrow \mathbb{R}, k=1,2, \ldots, n$, the random vector

$$
\left(f_{1}\left(X\left(I_{1}\right)\right), f_{2}\left(X\left(I_{2}\right)\right), \ldots, f_{n}\left(X\left(I_{n}\right)\right)\right)
$$

consists of weakly associated random variables.

The next definition can be found in [2].

Definition 4.3. A family $\mathbb{X}=\left\{X_{i}, i \in I\right\}$ is called positively associated if

$$
\operatorname{cov}\left(g\left(X\left(A_{g}\right)\right), h\left(X\left(A_{h}\right)\right)\right) \geq 0
$$

for any disjoint sets $A_{g}, A_{h} \subseteq I$, and all nondecreasing functions $g: \mathbb{R}^{\left|A_{g}\right|} \rightarrow \mathbb{R}$ and $h: \mathbb{R}^{\left|A_{h}\right|} \rightarrow \mathbb{R}$.

Clearly, for families of random variables $(d=1)$, the notions of weak association and positive association coincide. It is interesting that, owing to this coincidence, the notions of weak association between blocks and positive association between blocks are also the same. In fact, a definition for the latter should look as follows.

Definition 4.4. A family $\mathbb{X}$ is called positively associated between blocks if, for all nondecreasing functions $f_{k}: \mathbb{R}^{\left|I_{k}\right|} \rightarrow \mathbb{R}, k=1,2, \ldots, n$, the vector

$$
\left(f_{1}\left(X\left(I_{1}\right)\right), f_{2}\left(X\left(I_{2}\right)\right), \ldots, f_{n}\left(X\left(I_{n}\right)\right)\right)
$$

is positively associated, i.e. for any disjoint finite sets $A_{g}, A_{h} \subset\{1,2, \ldots, n\}$, and any nondecreasing functions $g: \mathbb{R}^{\left|A_{g}\right|} \rightarrow \mathbb{R}$ and $h: \mathbb{R}^{\left|A_{h}\right|} \rightarrow \mathbb{R}$,

$$
\operatorname{cov}\left(g\left(f_{i}\left(X\left(I_{i}\right)\right), i \in A_{g}\right), h\left(f_{j}\left(X\left(I_{j}\right)\right), j \in A_{h}\right)\right) \geq 0,
$$

if the covariance exists. 
We see that both (4.1) and Definition 4.2 state that the random variables $f_{1}\left(X\left(I_{1}\right)\right)$, $f_{2}\left(X\left(I_{2}\right)\right), \ldots, f_{n}\left(X\left(I_{n}\right)\right)$ are weakly associated, so there is no need to define positive association between blocks.

Remark 4.1. It is easy to see that, for jointly Gaussian random variables, the two types of relaxed association considered in the present paper (association between blocks and weak association between blocks) coincide and are equivalent to nonnegativity of covariances of random variables which are not in the same block.

Next we shall give a formal statement of the original form and a relaxed form of negative association due to Joag-Dev and Proschan [8].

Definition 4.5. A family $\mathbb{X}=\left\{X_{i}, i \in I\right\}$ is called negatively associated if

$$
\operatorname{cov}\left(g\left(X\left(A_{g}\right)\right), h\left(X\left(A_{h}\right)\right)\right) \leq 0
$$

for any disjoint sets $A_{g}, A_{h} \subseteq I$, and all nondecreasing functions $g: \mathbb{R}^{\left|A_{g}\right|} \rightarrow \mathbb{R}$ and $h: \mathbb{R}^{\left|A_{h}\right|} \rightarrow \mathbb{R}$.

Definition 4.6. A family $\mathbb{X}$ is called negatively associated between blocks if, for all nondecreasing functions $f_{k}: \mathbb{R}^{\left|I_{k}\right|} \rightarrow \mathbb{R}, k=1,2, \ldots, n$, the vector

$$
\left(f_{1}\left(X_{I_{1}}\right), f_{2}\left(X_{I_{2}}\right), \ldots, f_{n}\left(X_{I_{n}}\right)\right)
$$

is negatively associated, i.e. for any disjoint finite sets $A_{g}, A_{h} \subset\{1,2, \ldots, n\}$, and any nondecreasing functions $g: \mathbb{R}^{\left|A_{g}\right|} \rightarrow \mathbb{R}$ and $h: \mathbb{R}^{\left|A_{h}\right|} \rightarrow \mathbb{R}$,

$$
\operatorname{cov}\left(g\left(f_{i}\left(X_{I_{i}}\right), i \in A_{g}\right), h\left(f_{j}\left(X_{I_{j}}\right), j \in A_{h}\right)\right) \leq 0,
$$

if the covariance exists.

We conclude this section with a definition of the corresponding notions for increments of processes.

Definition 4.7. A $d$-dimensional stochastic process $\left\{X_{t}, t \geq 0\right\}$ has block weakly associated or block negatively associated increments if, for every $n \in \mathbb{N}$ and any choice of $0<t_{1}<t_{2}<$ $\cdots<t_{n}$, the increments

$$
X_{t_{1}}-X_{0}, \quad X_{t_{2}}-X_{t_{1}}, \quad \ldots, \quad X_{t_{n}}-X_{t_{n-1}}
$$

form the sequence of vectors which are weakly or, respectively, negatively associated between blocks formed by the $d$ components of each increment $X_{t_{i}}-X_{t_{i-1}}$.

\section{Limit theorems under weak association between blocks}

Let $X_{1}, X_{2}, \ldots$ be a sequence of $d$-dimensional random vectors. After building blocks upon the coordinates of consecutive vectors we may compare the notions of weak association of random vectors $\left\{X_{k}\right\}$ (Definition 4.1) and weak association between blocks (Definition 4.2). Formally, the latter is weaker: in place of nondecreasing functions $g$ and $h$ 'directly' acting on vectors, i.e.

$$
g\left(X_{\pi(1)}, X_{\pi(2)}, \ldots, X_{\pi(k)}\right), \quad h\left(X_{\pi(k+1)}, X_{\pi(k+2)}, \ldots, X_{\pi(m)}\right),
$$


the latter definition operates with factorizations, i.e.

$$
g\left(f_{\pi(1)}\left(X_{\pi(1)}\right), \ldots, f_{\pi(k)}\left(X_{\pi(k)}\right)\right), \quad h\left(f_{\pi(k+1)}\left(X_{\pi(k+1)}\right), \ldots, f_{\pi(m)}\left(X_{\pi(m)}\right)\right) .
$$

As already mentioned in the introduction, we are not able to exhibit any example of a sequence $\left\{X_{k}\right\}$ which is weakly associated between blocks, but not weakly associated. On the other hand, the computations performed in Section 2 and based on the covariance interpolation formula suggest that it might be a serious advantage to deal with factorized functions while checking whether the sequence is weakly associated between blocks. This is one reason for including the present section into the paper.

The other reason is that the complete generalization of Newman's central limit theorem [14] and Newman-Wright's invariance principle [16] for sums of stationary associated random variables, originally proved by Burton et al. [3] for weakly associated random vectors, remains valid under weak association between blocks, without any change in its proof. Here 'complete generalization' means including as a particular case the central limit theorem for independent and identically distributed random vectors, with covariance matrices possibly containing negative entries.

Theorem 5.1. Let $X_{1}, X_{2}, \ldots$ be a strictly stationary sequence of d-dimensional random vectors, which are weakly associated between blocks, and let $S_{n}=X_{1}+X_{2}+\cdots+X_{n}$.

If $\mathrm{E} X_{1}=0, \mathrm{E}\left\|X_{1}\right\|^{2}<+\infty$, and $\sum_{j=2}^{\infty} \mathrm{E} X_{1}^{k} X_{j}^{l}<+\infty$ for all $k, l=1, \ldots, d$ (where $X_{j}^{k}$ is the kth component of the vector $X_{j}$ ), then

$$
\frac{S_{n}}{\sqrt{n}} \stackrel{\mathrm{D}}{\rightarrow} \mathcal{N}(0, \Sigma) \quad \text { as } n \rightarrow \infty,
$$

where $\Sigma=\left(\sigma_{k l}\right)_{k, l=1 \ldots, d}$ and $\sigma_{k l}=\mathrm{E} X_{1}^{k} X_{1}^{l}+2 \sum_{j=2}^{\infty} \mathrm{E} X_{1}^{k} X_{j}^{l}$.

Moreover, if

$$
Y_{n}(t)=\frac{1}{\sqrt{n}} S_{[n t]}, \quad t \in \mathbb{R}^{+}
$$

(or $Y_{n}(t)$ is a polygonal interpolation between points $\left.\left(k / n, S_{k} / \sqrt{n}\right)\right)$, then

$$
Y_{n} \stackrel{\mathrm{D}}{\rightarrow} W_{\Sigma} \text { as } n \rightarrow \infty
$$

on the function space $C\left(\mathbb{R}^{+}: \mathbb{R}^{d}\right)$, where $W_{\Sigma}$ is a Wiener process with covariance matrix $\Sigma$.

Proof. In their proof, Burton et al. [3] used the weak association of the random variables

$$
f_{j}\left(X_{j}\right)=\left\langle a_{j}, X_{j}\right\rangle=\sum_{k=1}^{d} a_{j}^{k} X_{j}^{k},
$$

where $a_{j}^{1}, a_{j}^{2}, \ldots, a_{j}^{d} \geq 0$ are suitably chosen (for tightness purposes, convergence of finitedimensional distributions, etc.). Our assumption on weak association between blocks provides exactly the same information.

Remark 5.1. It is also likely that other existing limit theorems for associated random variables (see, e.g. [2, Chapter 3]) can be proved under relaxed assumptions like weak association between blocks and in a similar way as Theorem 5.1. In particular, work is in progress towards results on convergence to stable laws with infinite variance, paralleling [4]. 


\section{Acknowledgement}

During the preparation of this paper we had fruitful discussions with Tomasz Schreiber. He passed away on 1 December 2010. We dedicate this paper to his memory.

\section{References}

[1] Barlow, R. E. And Proschan, F. (1996). Mathematical Theory of Reliability. Society for Industrial and Applied Mathematics, Philadelphia, PA.

[2] Bulinski, A. And Shashkin, A. (2007). Limit Theorems for Associated Random Fields and Related Systems. World Scientific, Hackensack, NJ.

[3] Burton, R. M., Dabrowski, A. R. and Dehling, H. (1986). An invariance principle for weakly associated random vectors. Stoch. Process. Appl. 23, 301-306.

[4] Dabrowski, A. R. And Jakubowski, A. (1994). Stable limits for associated random variables. Ann. Prob. 22, $1-16$.

[5] Esary, J. D., Proschan, F. And Walkup, D. W. (1967). Association of random variables, with applications. Ann. Math. Statist. 38, 1466-1474.

[6] Glasserman, P. (1992). Processes with associated increments. J. Appl. Prob. 29, 313-333.

[7] Houdré, C., Pérez-Abreu, V. and Surgailis, D. (1998). Interpolation, correlation identities, and inequalities for infinitely divisible variables. J. Fourier Anal. Appl. 4, 651-668.

[8] Johg-Dev, K. and Proschan, F. (1983). Negative association of random variables, with applications. Ann. Statist. 11, 286-295.

[9] KarŁowska-Pik, J. And Schreiber, T. (2008). Association criteria for $M$-infinitely-divisible and $U$-infinitelydivisible random sets. Prob. Math. Statist. 28, 169-178.

[10] Liggett, T. M. (1985). Interacting Particle Systems. Springer, New York.

[11] Marshall, A. W. AND Olkin, I. (1979). Inequalities: Theory of Majorization and Its Applications. Academic Press, New York.

[12] Maruyama, G. (1970). Infinitely divisible processes. Teor. Verojat. Primen. 15, 3-23 (in Russian). English translation: Theory Prob. Appl. 15, 1-22.

[13] Müller, A. And Stoyan, D. (2002). Comparison Methods for Stochastic Models and Risks. John Wiley, Chichester.

[14] Newman, C. M. (1980). Normal fluctuations and the FKG inequalities. Commun. Math. Phys. 74, 119-128.

[15] Newman, C. M. (1983). A general central limit theorem for FKG systems. Commun. Math. Phys. 91, 75-80.

[16] Newman C. M., ANd Wright, A. L. (1981). An invariance principle for certain dependent sequences. Ann. Prob. 9, 671-675.

[17] Pitt, L. D. (1982). Positively correlated normal variables are associated. Ann. Prob. 10, 496-499.

[18] Rajput, B. S. AND Rosiński, J. (1989). Spectral representations of infinitely divisible processes. Prob. Theory Relat. Fields 82, 451-487.

[19] ReSNICK, S. I. (1988). Association and multivariate extreme value distributions. In: Studies in Statistical Modelling and Statistical Science, ed. C. C. Heyde, Statistical Society of Australia, pp. 261-271.

[20] Samorodnitsky, G. (1995). Association of infintely divisible random vectors. Stoch. Process. Appl. 55, 45-55.

[21] Sato, K.-I. (1999). Lévy Processes and Infinitely Divisible Distributions. Cambridge University Press, Cambridge. 